\title{
A construção da experiência omnichannel de relacionamento das interfaces do Sistema Sebrae Omnichannel experience design of Sebrae System's interfaces
}

\author{
Henrique Teles da Mota \& Tiago Barros Pontes e Silva
}

experiência do usuário, omnichannel, omnicanalidade, Sebrae

\begin{abstract}
A crescente incorporação de tecnologias móveis de comunicação à nossa rotina vem estimulando o surgimento de novos comportamentos. $\mathrm{O}$ acesso à internet, antes restrito a computadores de mesa, tem se tornado cada vez mais pervasivo, complementando - ou concorrendo com - a nossa experiência de vida no mundo material. Dessa forma, se faz necessário repensar a maneira como projetar interfaces que respondam a esses novos contextos e a essas novas necessidades, estruturando ambientes que integrem os diversos canais por meio dos quais consumimos informações, produtos e serviços. Esse cenário de máxima integração é deniminado de omnichannel, ou omnicanalidade, e o presente trabalho objetiva identificar quais as contribuições que as abordagens de design de interfaces interativas apresentam para a sua materialização, visando investigar a aplicação prática do uso dessas abordagens no redesenho dos canais de relacionamento do sistema Sebrae. O estudo foi organizado em dois blocos que, combinados, viabilizaram as conclusões alcançadas nesse trabalho. O primeiro bloco contemplou a revisão bibliográfica e análise dos documentos relativos à conformação do Sistema Sebrae. O segundo bloco tratou do estudo do caso de integração dos canais de relacionamento do Sistema Sebrae, por meio questionário, entrevistas e análises de interface. Foi possível, assim, identificar que o processo parte de definições estratégicas genéricas e segue por etapas de especificação que culminam na materialização de interfaces. Todavia, também foi possível identificar que projetos de integração de canais com a dimensão e complexidade do Sebrae apresentam desafios de execução que devem ser discutidos nas abordagens de design, como o exemplo de desafios relacionados à cultura organizacional.
\end{abstract}

user experience, omnichannel, omnicanality, Sebrae

The increasing incorporation of mobile communication technologies into our routine has been a stimulus for the emergence of new behaviors and access to the internet, once restricted to desktop computers, has become increasingly pervasive, complementing - or competing with - our life experience in the physical world. In this way, it is necessary to rethink the way to design interfaces that respond to these new contexts and these new needs, structuring environments that integrate the various channels through which we consume information, products and services. This scenario of maximum integration is referred to as omnichannel, and the present work aims to identify the contributions the interactive interfaces' design approaches present for its materialization in the context of the Sebrae System. The study was organized in two stages. The first contemplated the bibliographic review and analysis of the documents related to the conformation of the Sebrae System. The second was a case study of the integration of the channels of the Sebrae System, through questionnaire, interviews and interface analysis. It was thus possible to identify that the process starts from generic strategic definitions and follows by specification stages that culminate in the materialization of interfaces. However, it has also been possible to identify that channel integration projects with the size and complexity of the Sebrae present implementation challenges that should be discussed in design approaches, such as the example of organizational culture challenges.

\section{Introdução}

Embora seja difícil precisar os efeitos do uso da internet em nosso comportamento, é inegável que sua incorporação à nossa rotina modificou profundamente a forma como nos relacionamos com a tecnologia e entre nós mesmos. Desde o seu surgimento, no final da década de 1960, a maneira como compartilhamos e consumimos informação sofreu grandes transformações, e o surgimento e a popularização dos smartphones nas duas primeiras décadas dos anos 2000 parecem catalisar esse processo.

Anais do 9 CIDI e 9 CONGIC

Luciane Maria Fadel, Carla Spinillo, Anderson Horta, Cristina Portugal (orgs.)

Sociedade Brasileira de Design da Informação - SBDI

Belo Horizonte | Brasil | 2019

ISBN $978-85-212-1728-2$
Proceedings of the 9th CIDI and 9th CONGIC

Luciane Maria Fadel, Carla Spinillo, Anderson Horta, Cristina Portugal (orgs.)

Sociedade Brasileira de Design da Informação - SBDI Belo Horizonte | Brazil | 2019

ISBN 978-85-212-1728-2 
Segundo dados do Cetic - Centro Regional de Estudos para o Desenvolvimento da Sociedade da Informação, 106 milhões de brasileiros já estavam conectados à internet em 2017 , e $96 \%$ desse grupo o fazia por meio do celular. Isso se traduz em pouco mais da metade da população com mobilidade no acesso à internet, o que desperta o potencial de desenvolvimento de uma série de novos comportamentos, em diferentes lugares e também de maneira complementar a diversas atividades.

No que diz respeito ao comportamento do consumidor, os impactos dessas transformações já vêm sendo observados por empresas e instituições de diversos setores. Surge o que se chama de consumidor omnichannel, que seria aquele que faz uso de diversos canais de relacionamento para a conclusão de sua jornada de consumo - ele pesquisa preços e atributos dos produtos e serviços no ambiente digital para efetuar a compra nas lojas físicas ou então conhece o produto no ambiente físico para depois efetuar sua compra no ambiente online.

Tal cenário tem impactos diretos na forma como os canais de relacionamento que atendem esse público são estruturados e apresentados. Assim, esse fenômeno desperta a necessidade de identificar métodos para o desenvolvimento de uma estrutura omnichannel de relacionamento, prevendo não somente a funcionalidade de cada canal de forma autônoma e isolada, mas também a sua atuação integrada ao ecossistema de canais em que está inserido.

Portanto, o presente estudo tem como objetivo investigar o desenvolvimento de uma arquitetura omnichannel de relacionamento a partir da perspectiva do design de interfaces, visando estudar a aplicação prática do uso dessas abordagens no redesenho dos canais de relacionamento do sistema Sebrae, segundo a diretriz estratégica estabelecida pelo Sebrae Nacional.

\section{Canais de relacionamento}

A proposição de uma arquitetura integrada de canais de relacionamento apresenta como ponto de partida a própria definição de canal. Fazendo uma analogia com os conceitos de canais de marketing e canais de distribuição, podemos entender canais de relacionamento como o conjunto de organizações interdependentes envolvidas na distribuição e disponibilização de produtos e serviços (Coughlan et al., 2012). São os pontos de contato entre empresa e consumidor ou os meios pelos quais a interação entre eles acontece (Neslin et al. 2006, p. 96). Canais seriam, por exemplo, uma loja física, um site, uma central de relacionamento. Neles são distribuídos produtos, serviços e informações e por meio deles uma empresa desenvolve relacionamento com seus consumidores (Mehta et al. 2002).

Nesse sentido, uma estratégia de canais é a proposta de como esses canais se organizam e contribuem para o cumprimento dos objetivos corporativos (Mehta et al. 2002). A estratégia de canais define a forma como cada um dos canais interage entre si e com o público final, e quais funções cada um deles assume nesse ecossistema. Os canais podem estabelecer uma relação direta com o consumidor final ou então serem intermediados por atacadistas, varejistas ou ambos, o que interfere não somente na autonomia e controle da distribuição de produtos e prestação de serviços de uma empresa, mas também nos custos de operação e logística que decorrem desses arranjos (Kotler et al. 2015).

Sua organização compõe um elemento importante da entrega de valor para o cliente, uma vez que é por meio deles que o consumo é efetivado de fato. Dessa forma, é importante que a definição dos objetivos de cada canal tenha como foco de atenção as necessidade e expectativas do público que consome por meio dele, além, evidentemente, de considerar a natureza da empresa e dos produtos e serviços que oferta (Kotler et al. 2015). A forma como a rede de canais se configura caracteriza os diferentes tipos de arquitetura de canais que uma empresa pode adotar, podendo ele ser single, multi, cross ou omnichannel.

\section{Do single ao omnichannel}

A configuração mais simples de arquitetura de canais é aquela em que figura apenas um canal de relacionamento, o chamado single channel. Nesse caso, não há que se falar em integração 
ou interação, pois o consumidor só tem uma única possibilidade de acessar os serviços ou produtos de uma empresa. Esse é o típico caso das empresas que possuem um ponto físico de atendimento - loja, quiosque, banca - e não possuem nenhum outro canal.

Quando existem outros canais, mas pouca ou nenhuma interação entre eles, trata-se de uma arquitetura multichannel. Nesses casos, existe mais de um ponto de contato e mais de uma via de acesso entre consumidor e empresa. Entretanto, cada uma dessas vias opera de maneira independente e a própria gestão dos canais é orientada com esse foco, de maneira a identificar o desempenho de cada canal isoladamente (Beck \& Rygl 2015; Verhoef et al. 2015).

Com o aumento da integração entre os canais, configura-se a chamada arquitetura crosschannel. Nesse caso, existe uma integração parcial de uma série de canais de relacionamento e existe a possibilidade de um consumidor transitar entre eles, inclusive no que diz respeito à prestação de serviços (Beck \& Rygl, 2015; Verhoef et al., 2015). Por exemplo, é possível que um consumidor troque na loja física um produto adquirido na internet ou então que utilize um voucher de desconto que recebeu no seu e-mail na prestação de um serviço presencial. Entretanto, vale ressaltar que diversos autores que abordam o assunto omitem a instância crosschannel e abordam apenas os casos de multi e omnichannel. Nesses casos, as características aqui apresentadas são atribuídas ao modelo omnichannel.

Por fim, existe o que é considerado o ápice da integração entre os canais, o omnichannel. Nesse caso, os próprios limites entre cada um dos canais tornam-se indefinidos e a navegação entre eles se torna completamente fluida. Tal comportamento passa a ser não somente previsto, como estimulado (Piotrowicz \& Cuthbertson, 2014; Verhoef et al., 2015).

Considerando o contexto de omnicanalidade e a expectativa de uma experiência de consumo fluida entre os diversos canais de relacionamento de uma empresa, esse mesmo serviço deveria poder ser acessado e consumido em qualquer um dos canais disponibilizados, permitindo ainda ao usuário uma navegação consistente e harmônica entre eles. Por exemplo, um aluno que saísse mais cedo de uma aula presencial poderia continuar o consumo do curso do momento em que parou no seu celular no caminho de casa e, eventualmente, terminá-lo no computador quando chegasse.

Essa narrativa, no entanto, apresenta uma complexidade em sua construção inversamente proporcional à simplicidade que se intenciona apresentar ao usuário e não se limita a uma simples transposição de um mesmo conteúdo em diversos meios. A construção de uma linearidade transversal a diversos formatos de canais de relacionamento apresenta não só desafios tecnológicos - muito antes disso, é preciso identificar o fio condutor que garante a coerência de uma jornada fluida, uma narrativa coerentemente concatenada.

\section{Método}

A pesquisa foi organizada em dois blocos. O primeiro (Figura 1) constituiu na análise documental, que compreendeu a revisão bibliográfica e revisão dos documentos relativos à estrutura do Sistema Sebrae. A revisão bibliográfica contemplou temas relacionados a abordagens de design de interfaces interativas, experiência do usuário, canais de relacionamento e narrativa omnichannel. O objetivo dessa etapa foi identificar as propostas teóricas já desenvolvidas em cada uma dessas áreas, como forma de apresentar os conceitos abordados nessa investigação e de referenciar as abordagens de design de interfaces interativas para, posteriormente, validar de que forma podem contribuir para a construção de uma experiência omnichannel.

A revisão dos documentos relativos à estrutura do Sistema Sebrae, por sua vez, serviu para contextualizar o objeto de análise em que ocorreu a investigação empírica - o Sistema Sebrae em si. Por meio dessa análise, foi possível apresentar a organização estrutural do sistema e as características que podem influenciar o processo de transformação de sua estrutura de canais de relacionamento. Nessa etapa, foi possível recuperar projetos em andamento e compreender, em certa medida, a lógica empregada para a integração dos canais em uma estrutura omnichannel. 
Figura 1: Bloco 1 de pesquisa, contemplando a Revisão Bibliográfica e as Características do Sistema Sebrae, obtidos por meio de Análise Documental.

\section{Bloco 1 - Análise Documental}

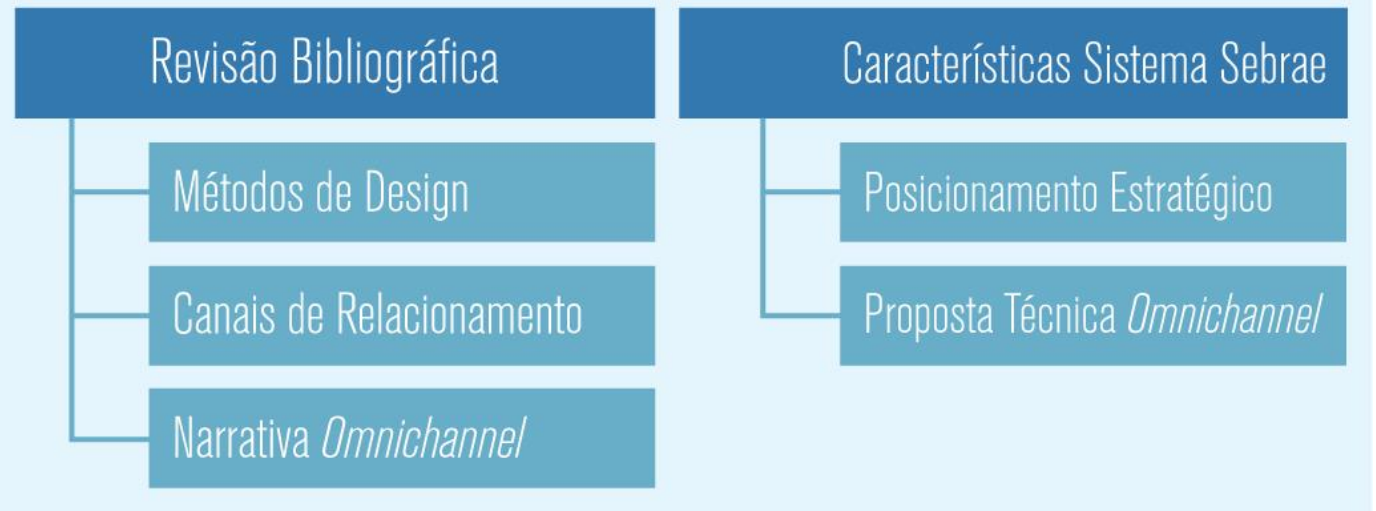

O segundo bloco (Figura 2) concentrou o estudo do caso de integração dos canais de relacionamento do Sistema Sebrae, a partir de um questionário, entrevistas realizadas com os atores envolvidos na gestão dos canais de relacionamento do Sistema Sebrae e análises de interface feitas a partir do acesso direto aos canais de relacionamento digitais. Ainda, ao final do processo de análise interno, um especialista em design de experiências integradas foi consultado para contribuir com uma visão técnica a respeito do processo.

Figura 2: Bloco 2 de pesquisa, contemplando a conformação atual dos canais e a coleta de percepções sobre o processo de integração.

\section{Bloco 2 - Análise Empírica}

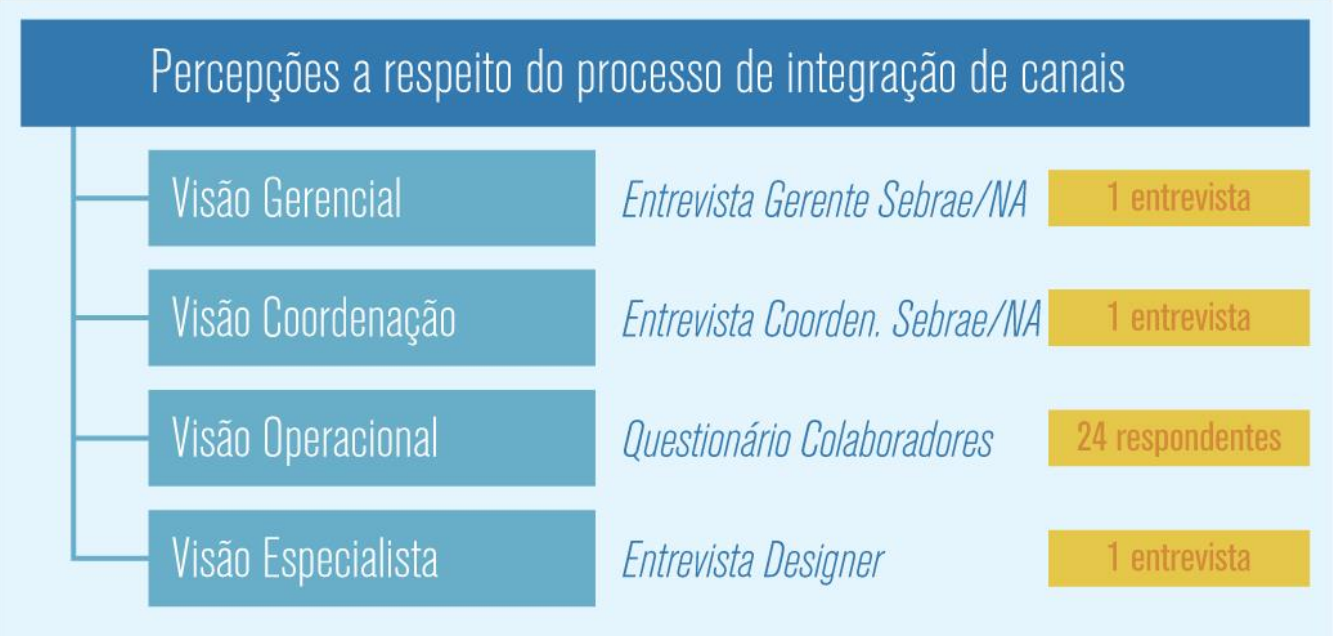

Quanto às análises realizadas dentro do Sistema Sebrae, a etapa seguiu uma progressão lógica desde a visão estratégica da empresa até a visão operacional, de maneira a explorar os diferentes pontos de vista de um processo de integração de canais de relacionamento a partir do espaço ocupacional de cada colaborador. 
Sendo assim, primeiramente, foi entrevistada a então gerente da Unidade de Relacionamento do Sebrae Nacional, seguida pela então gestora responsável pela coordenação da relação entre o Sebrae Nacional e as sedes estaduais do Sebrae e, por fim, um questionário foi aplicado para coletar informações dos colaboradores envolvidos diretamente com a operação. Dessa forma, foi possível construir um entendimento progressivo, desde a concepção estratégica de uma integração de canais de relacionamento, até à visão dos responsáveis por essa operação na prática.

Foi também analisado o estado atual e forma de funcionamento dos canais de relacionamento, o que ocorreu por meio de entrevistas com os gestores estaduais dos canais Agência e Central de Relacionamento e análise dos ambientes digitais do próprio Sebrae Nacional e de cada estado.

\section{O Sistema Sebrae}

O Serviço Brasileiro de Apoio às Micro e Pequenas Empresas (Sebrae) é uma entidade privada sem fins lucrativos que opera prioritariamente com recursos públicos para promover a competividade e o desenvolvimento sustentável dos pequenos negócios e estimular o empreendedorismo com o objetivo de fortalecer a economia nacional.

Sua atuação ocorre de maneira sistêmica e se estende por todo o território nacional por meio de canais de relacionamento digitais e remotos, pontos físicos de atendimentos distribuídos em cada um dos 26 estados e do Distrito Federal e uma sede nacional, localizada em Brasília. À sede nacional cabe a designação de Sebrae, e compete a ela a atividade de coordenação do sistema, enquanto as unidades estaduais, designadas coletivamente como Sebrae/UF, sendo UF a sigla da Unidade Federativa correspondente àquela unidade, se ocupam da atividade operacional. A esse conjunto, atribui-se a denominação de Sistema Sebrae.

\section{Proposta técnica para a omnicanalidade}

O desenvolvimento de uma proposta de omnicanalidade no Sebrae teve como premissa a estrutura existente de canais de relacionamento e a forma como os produtos e serviços da empresa estavam organizados. Dessa maneira, era necessário propor uma solução que fosse aderente ao cenário posto e não somente um ideal de omnicanalidade que respondesse apenas às expectativas estratégicas definidas.

O primeiro passo, portanto, para a elaboração de uma proposta técnica de arquitetura omnichannel de relacionamento no Sebrae foi o mapeamento das ações que eram performadas em cada canal até então. Essa identificação foi feita em seu nível mais abstrato, em analogia ao modelo proposto por Garret (2011), a estratégia. O objetivo era identificar funções que fossem cumpridas por cada canal, independente da interface ou processo responsável por realizá-la.

Para essa identificação, foram reunidos os gestores nacionais dos canais de relacionamento e cada um deles listou as funções desempenhadas por cada canal. Funções como identificação, apresentação/oferta de serviços, avaliação de atendimento e outras foram identificadas em cada um dos canais. Foi possível, dessa forma, compreender com a arquitetura de cada canal estava definida e começar a visualizar possível pontos de convergência entre eles.

As funções foram, então, agrupadas, e organizadas em blocos de funções análogas, que estabeleciam uma sequência lógica, ainda que genérica, da estrutura de canais de relacionamento do Sistema Sebrae (Figura 3). Um segundo diagrama associa cada função ao canal em que ela está presente (Figura 4). 
Figura 3: Arquitetura genérica de canais de relacionamento do Sebrae. Fonte: Documentos em rede da Unidade de Relacionamento com o Cliente. Acessado em: 10/05/2019.

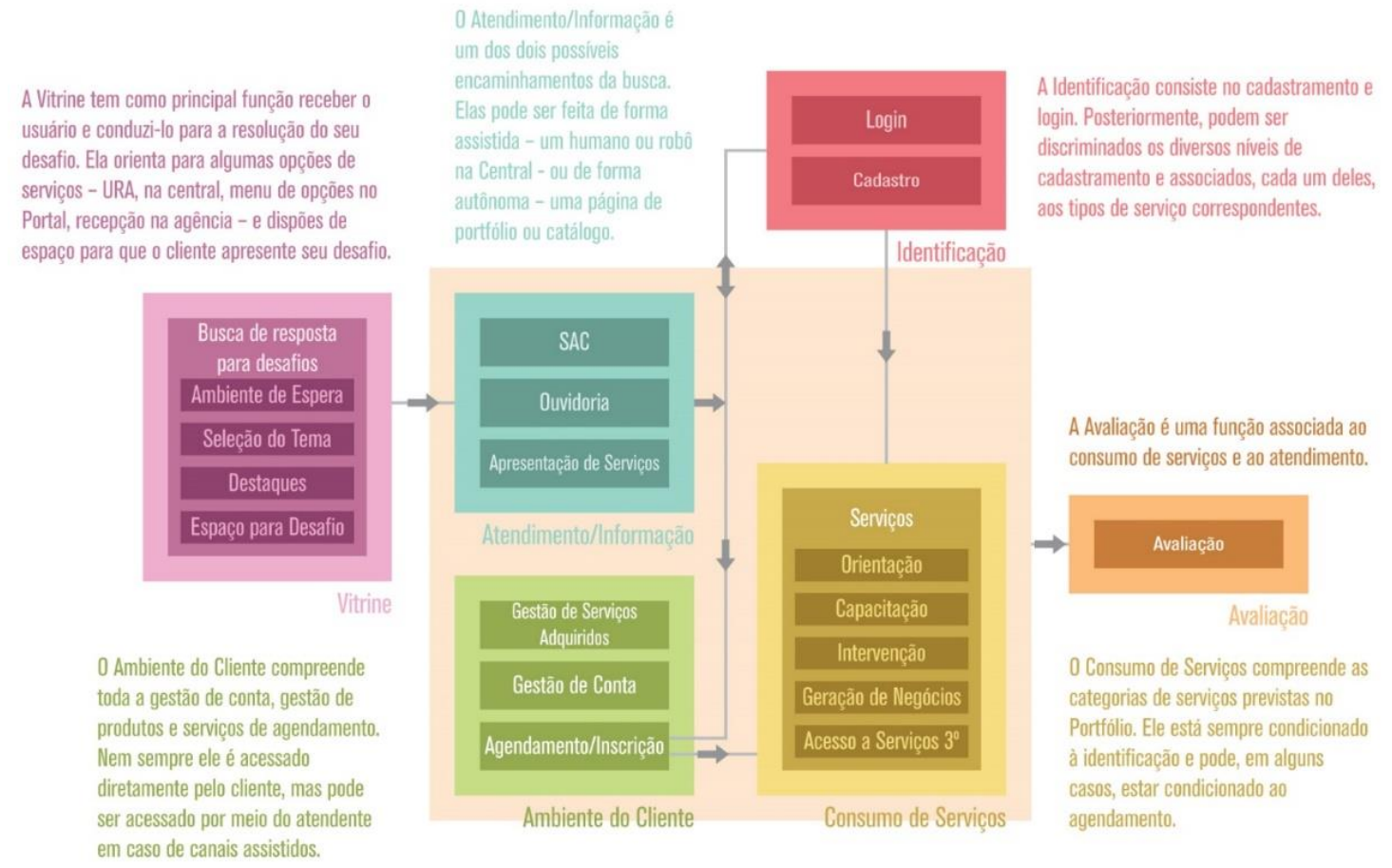

Figura 4: Arquitetura genérica com indicação dos canais relacionados. Fonte: Documentos em rede da Unidade de Relacionamento com o Cliente. Acessado em: 10/05/2019.

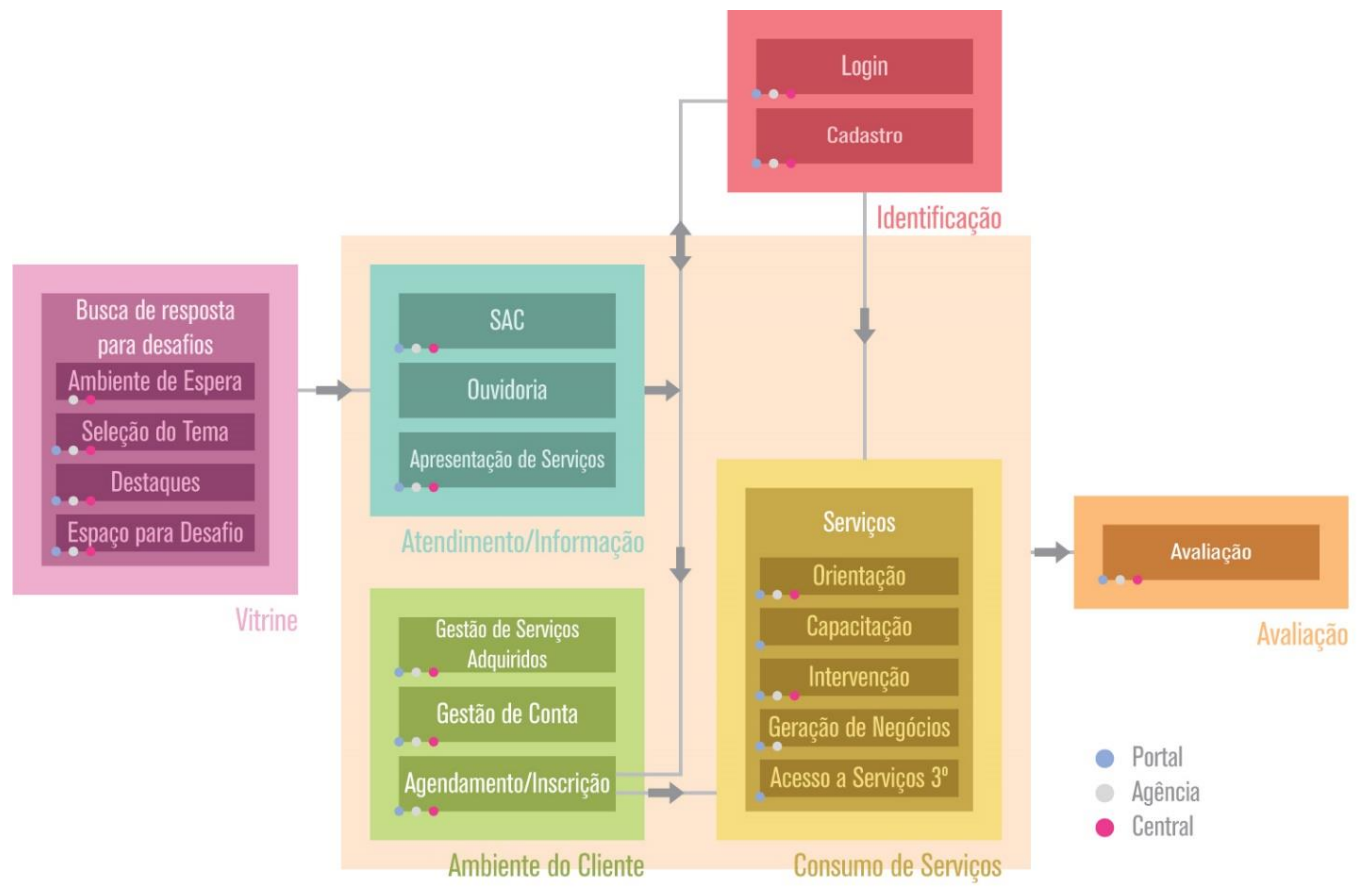

Anais do 9o Congresso Internacional de Design da Informação | CIDI 2019 
Foi possível visualizar, então, os pontos de intersecção entre os canais de relacionamento, partindo de uma estrutura genérica que refletia o processo de atendimento que ocorria em cada um deles, independentemente das características que os individualizavam.

Identificou-se, por exemplo, que todos os canais realizavam, de uma forma ou de outra, a apresentação do portfólio de produtos e serviços do Sebrae, ainda que não fosse igualmente possível consumi-los em todos eles. Um cliente poderia ter acesso à agenda de cursos por meio de um atendimento realizado na central telefônica do Sebrae, mas só poderia consumir o serviço, de fato, na agência, no caso de cursos presenciais, ou no Portal, no caso de cursos a distância.

O passo seguinte a essa definição genérica foi a particularização do fluxo para a realidade específica de cada canal (Figuras 5, 6 e 7), estabelecendo uma apresentação macro de navegação. Nessa etapa, a arquitetura de cada canal foi definida correlacionando as funções já previstas na arquitetura macro à sua estrutura particular.

Já é possível estabelecer, com algum grau de abstração, uma analogia entre as abordagens já conhecidas de design de interfaces interativas (Garret, 2011) e o método utilizado para determinar a arquitetura dos canais de relacionamento apresentada. Em ambos os casos, parte-se de uma concepção abstrata, que se expressa nas propostas funcionais, e segue-se uma etapa de especificação que culmina no detalhamento da interface, de fato.

A proposta ainda serve de base para a especificação do sistema que deve suportar essa operação, uma vez que fica claro onde cada tipo de conteúdo ou serviço será consumido e quais as possibilidades de navegação disponíveis para o usuário. Nesse estado de desenvolvimento, a proposta de arquitetura de canais de relacionamento funciona também como um diagrama de suporte para decisões negociais, uma vez que facilita a visualização da estrutura de canal e viabiliza uma estruturação mais coordenada de todo o ecossistema.

Figura 5: Proposta de arquitetura do canal Central de Relacionamento. Fonte: Documentos em rede da Unidade de Relacionamento com o Cliente. Acessado em: 10/05/2019.

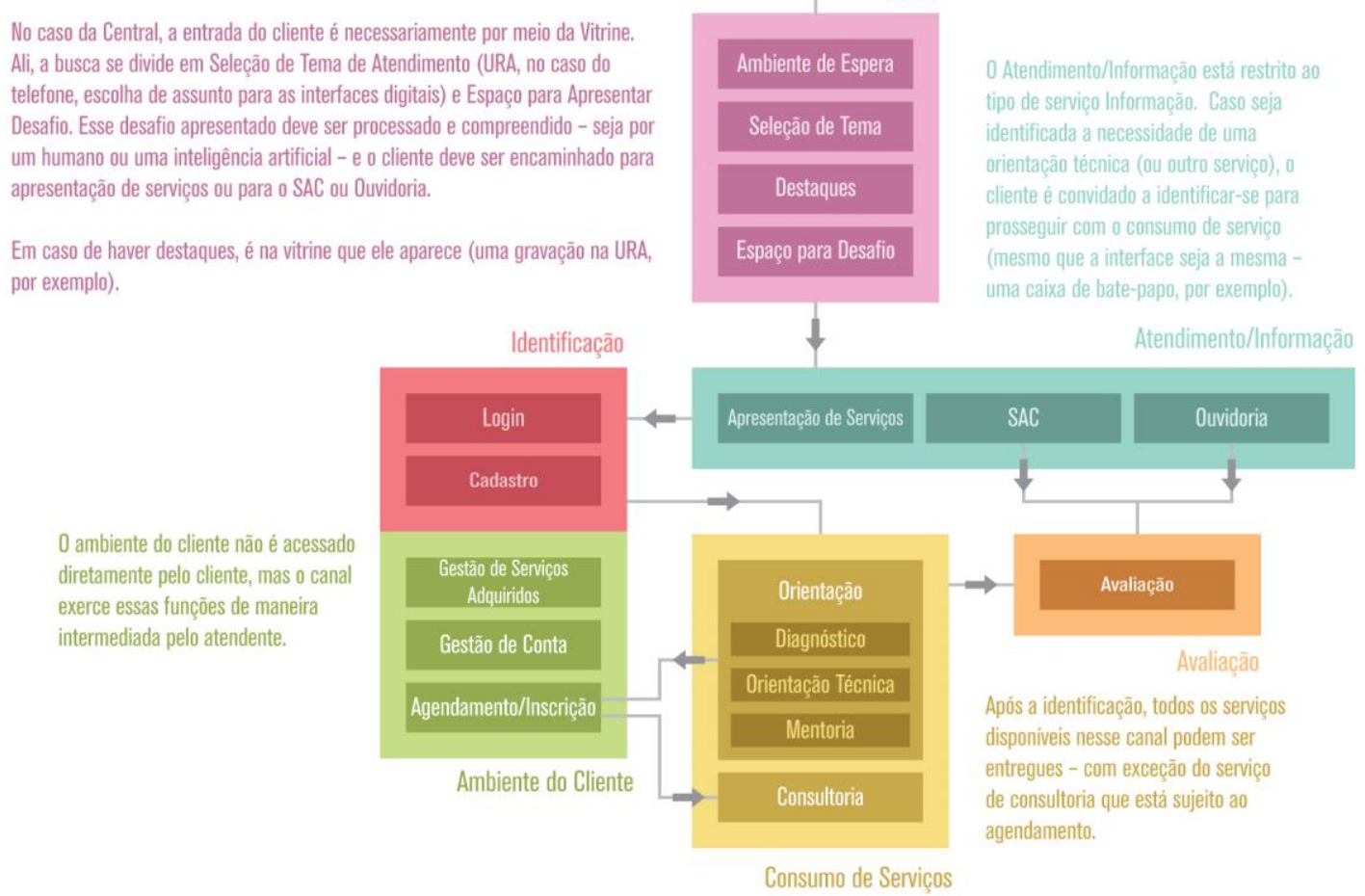


Figura 6: Proposta de arquitetura do canal Agência. Fonte: Documentos em rede da Unidade de Relacionamento com o Cliente. Acessado em: 10/05/2019.

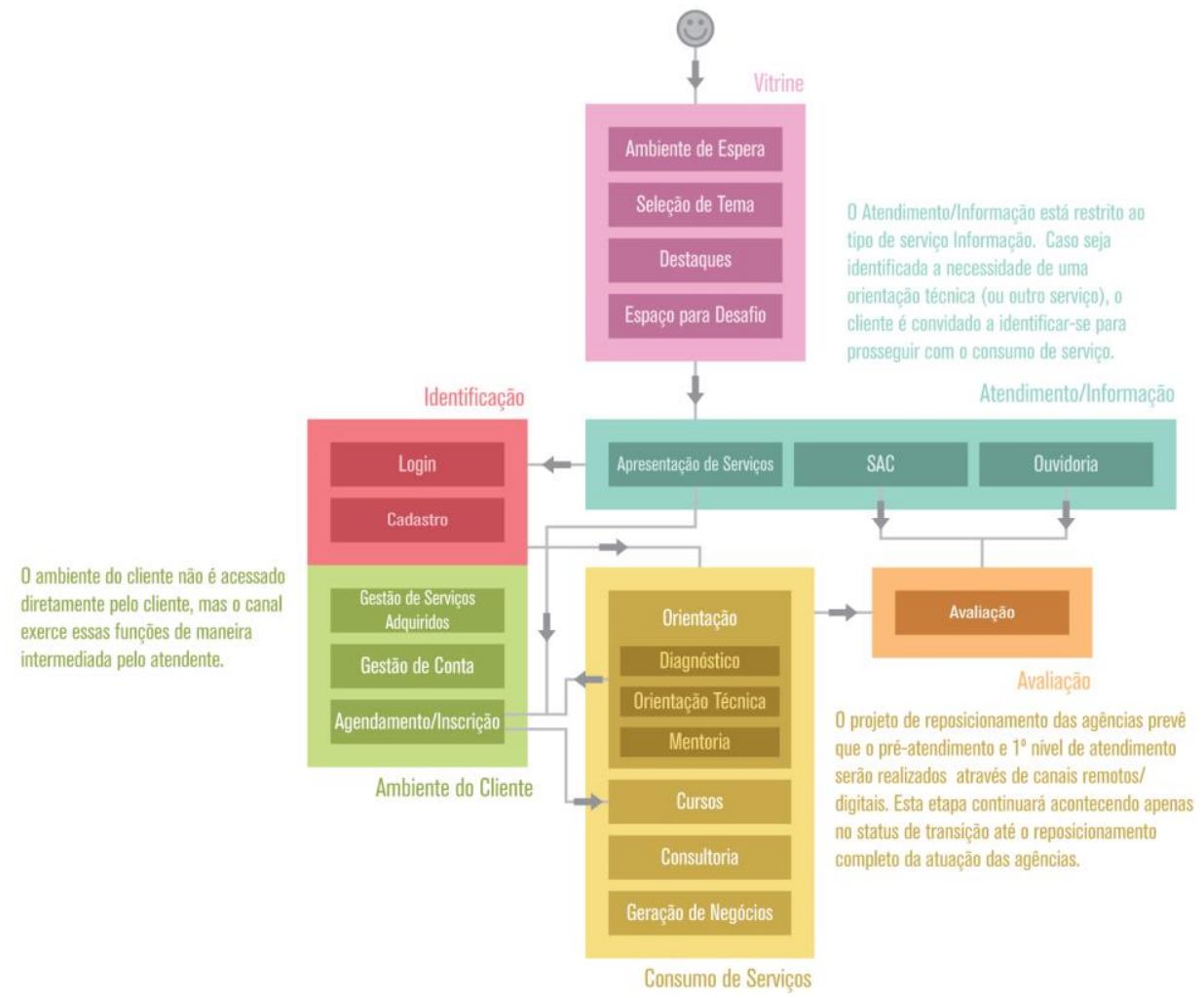

Figura 7: Proposta de arquitetura do canal Portal. Fonte: Documentos em rede da Unidade de Relacionamento com o Cliente. Acessado em: 10/05/2019.

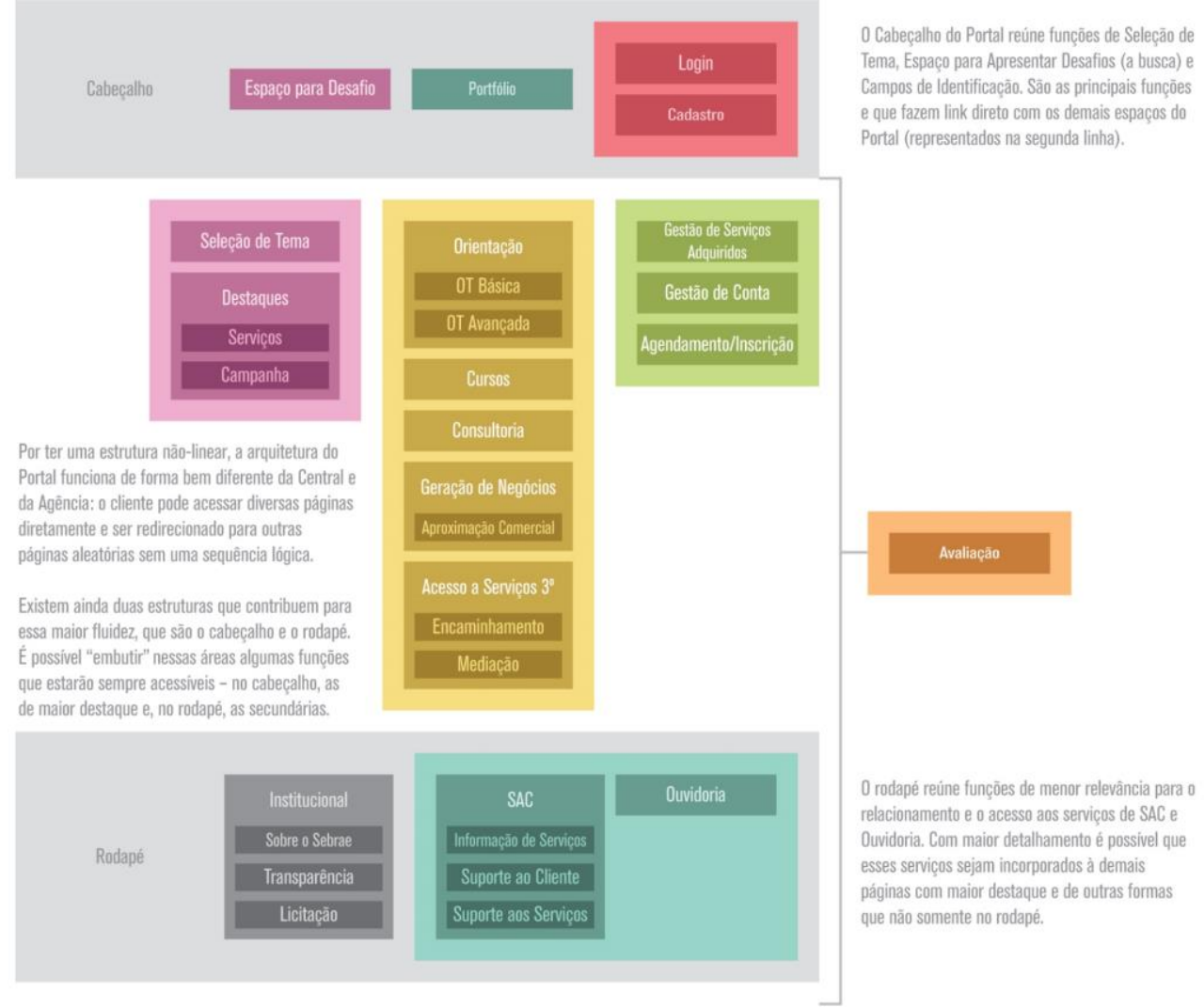

Anais do 9o Congresso Internacional de Design da Informação | CIDI 2019 
Todavia, sendo o canal de relacionamento a estrutura responsável pela distribuição dos produtos e serviços de uma empresa - os pontos de contato entre a empresa e o cliente (Coughlan et al., 2012) -, é natural que a sua organização envolva diretamente a organização da sua estrutura de portfólio.

\title{
5 Relato das percepções sobre o processo de integração de canais
}

Dado que a integração dos canais de atendimento e relacionamento de uma empresa influencia diretamente nos seus processos e na sua forma de funcionamento, o envolvimento dos colaboradores nesse tipo de proposta é essencial para viabilizar a sua execução.

Nesse sentido, é interessante observar como colaboradores envolvidos em diferentes etapas e setores da empresa podem ter visões contraditórias ou complementares a respeito do processo de integração de canais e como essas visões se relacionam com o diagnóstico do ecossistema de canais e, principalmente, com as abordagens de design de interfaces interativas.

Dessa forma, o ponto de partida dessa investigação foi, justamente, a própria definição de omnicanalidade. Para tanto, os respondentes do questionário ordenaram, dentre algumas opções pré-definidas, aquelas que mais caracterizavam essa integração, segundo o seu entendimento. $\mathrm{O}$ conceito de omnicanalidade foi, então, prioritariamente, associado à estrutura de sistemas única ou integrada, seguida de processos de atendimento únicos, base de conhecimento única, portfólio único e unidade estética (Figura 8).

Figura 8: O que significa ser omnichannel para os colaboradores do Sebrae.

\author{
Estrutura de sistemas única ou integrada \\ Processo de atendimento único ou integrado \\ Base de conhecimento única \\ Porttólio único \\ Unidade estética
}

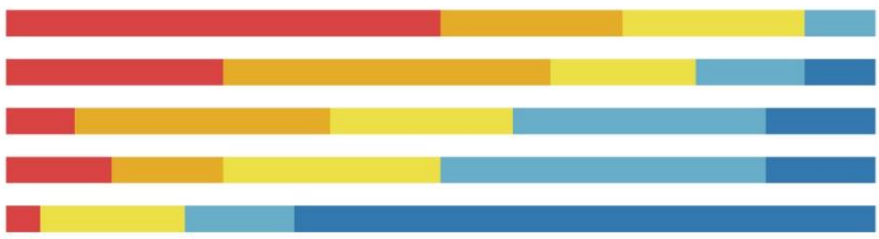

\section{Primeira escolha $\mathbf{\square} \mathbf{\square} \mathbf{u}$ Última escolha}

A indicação do questionário se complementa às informações coletadas nas entrevistas realizadas com a gerente e coordenadora, que associaram a omnicanalidade a uma estrutura integrada de base de dados, organização dos processos de atendimento, integração de portfólio e consolidação de uma cultura organizacional coerente.

Observa-se, portanto, certa coerência na percepção dos colaboradores, que, independente do espaço ocupacional na empresa, tendem a associar a omnicanalidade a processos e sistemas integrados.

Partindo dessa definição, ao serem questionados a respeito do grau de integração que percebiam entre os canais de atendimento e relacionamento do Sistema Sebrae, atribuindo uma nota de 0 a 10 a essa integração, sendo 0 nada integrados e 10, completamente integrados, a média de respostas foi 4,9, o que indica uma percepção de um sistema muito pouco integrado. Tal percepção é análoga à da gerente de relacionamento, que atribuiu uma nota 3 a esse grau de integração.

Assim, ainda que exista alinhamento a respeito do conceito omnichannel, o que ele caracteriza e o que seria necessário para alcançá-lo, existe também a percepção de que essa é uma realidade distante de ser alcançada, considerando o cenário atual do Sistema Sebrae. Uma possível justificativa para essa divergência pode estar relacionada à forma como os próprios colaboradores e gestores, de diferentes níveis hierárquicos lidam com esse processo. 
As questões relacionadas a isso foram identificadas como sendo relativas à cultura organizacional do Sebrae e foram recorrentes tanto nas entrevistas quanto nas respostas dos questionários, referenciando aspectos relacionados à alta diretoria e ao próprio corpo de colaboradores.

Ao tratar de possíveis obstáculos para a implementação de uma arquitetura omnichanne/ no Sebrae, foram feitas referências diretas à esfera estratégica da empresa. Alguns dos obstáculos apresentados no questionário, por exemplo, foram "Mudança de estratégia da instituição muito rapidamente, o que deixa as pessoas confusas e sem direção", "Decisão da diretoria" e "Falta de apoio e entendimento da alta direção".

Esse ponto também foi corroborado nas entrevistas - as definições estratégicas foram tomadas como sendo o ponto de partida desse processo, mas a falta de um posicionamento único e consistente também foi apontado como um potencial obstáculo.

Todavia, esse tipo de observação não se restringiu à porção estratégica da empresa e se estendeu também ao corpo de colaboradores. Assim, outros obstáculos apresentados foram "Comportamento e cultura das pessoas", "Planejamento em excesso, quase nada de execução" e até mesmo "Cultura ultrapassada". A gerente de relacionamento também corroborou essa ideia, ao afirmar que a cultura organizacional do Sebrae não favorece esse tipo de inovação e que existe certa resistência na equipe.

Nesse aspecto, torna-se evidente que as abordagens de design de interfaces, para além dos aspectos técnicos, devem considerar os aspectos culturais e comportamentais associados a eles. Em muitos contextos - talvez a maioria deles - as organizações não terão designers em seus mais altos cargos de gestão, ou mesmo no seu corpo de funcionários. Dessa forma, as abordagens de design devem considerar esses cenários e não se restringirem a realidades herméticas.

Na Figura 9 é possível identificar uma série de outros obstáculos que foram apontados pelos colaboradores. Outros aspectos que tiveram bastante destaque na lista foram 0 regionalismo e a infraestrutura tecnológica. O regionalismo refere-se, basicamente, à preocupação de que uma possível integração seja comprometida pela heterogeneidade de realidades no país. Já a infraestrutura tecnológica diz respeito aos riscos de os sistemas de atendimento e as bases de dados não serem capazes de suportar, de fato, a diversificada operação que ocorre em cada região.

Figura 9: Obstáculos para a integração dos canais segundo colaboradores.

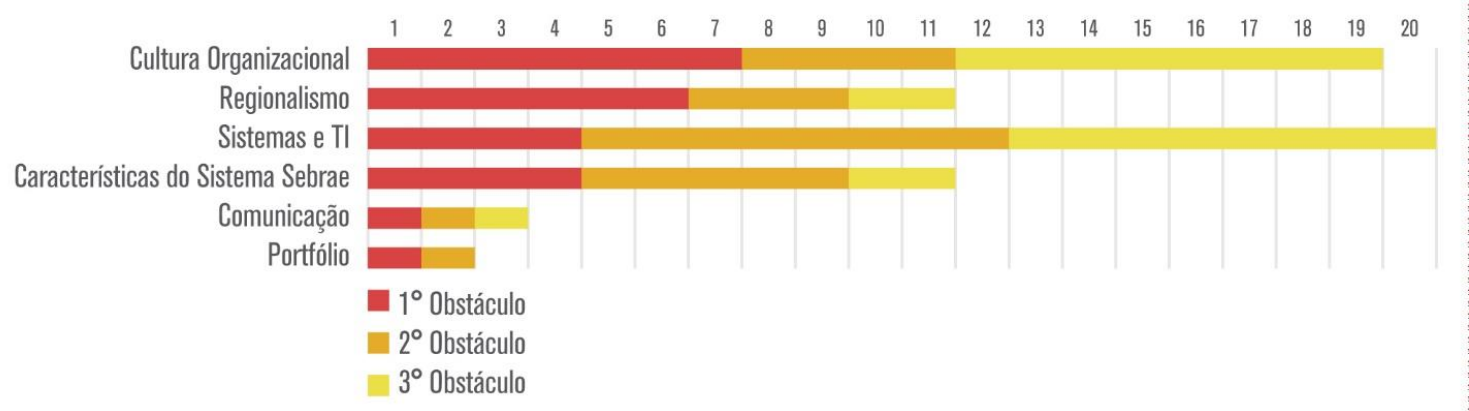

Analisando essas informações sob a ótica desse estudo, é possível estabelecer alguns paralelos com as abordagens de design de interfaces interativas. O regionalismo e a infraestrutura tecnológica, por exemplo, relacionam-se diretamente à capacidade de parametrização dos sistemas que suportam as interfaces e à definição dos seus requisitos. São questões plenamente contempladas no detalhamento de escopo de uma interface, por exemplo, quando são definidas as especificações funcionais e dos vários elementos que integram determinada estrutura (Garret, 2011). 
As questões categorizadas como "características do Sistema Sebrae", em muitos casos, referenciaram burocracias e processos internos da empresa, como "Contratos vigentes". Relacionam-se ao design de interfaces, de certa maneira, da mesma forma como as questões de cultura organizacional - não fazem parte do processo diretamente, mas compõem o contexto em que ele ocorre e, por isso, são relevantes.

"Comunicação" e "Portfólio" são referenciados, porém com pouca relevância. Nesse caso o interessante e observar que essa visão o que contraria a da coordenadora a esse respeito, que citou a organização do Portfólio como um dos pontos centrais no processo de integração dos canais.

Por fim, mesmo reconhecendo um baixo nível de integração e identificando uma série de obstáculos para alcançá-la, 21 entre os 24 respondentes consideram ser possível a implementação de uma arquitetura omnichanne/ de relacionamento no Sistema Sebrae e 16 apontam que já existem iniciativas nesse sentido nos seus estados, por meio de grupos de trabalho integrados, implantação de um modelo de atendimento comum ao Sistema Sebrae e utilização do mesmo sistema de atendimento, por exemplo.

Ao serem questionados sobre métodos específicos voltados para esse processo de integração, 8 respondentes afirmaram conhecê-los e referenciaram, genericamente, softwares e ferramentas que existem no mercado.

Diversas análises, portanto, podem ser feitas a partir da massa de dados geradas nessa etapa de pesquisa. Um primeiro ponto de que se destaca é a homogeneidade de entendimentos, mesmo em um contexto tão complexo e tão disperso como o Sistema Sebrae. O entendimento feito pela gerente e coordenadora converge com os colaboradores e gestores estaduais e isso já é uma vantagem para esse tipo de iniciativa.

Com relação aos obstáculos identificados - e com especial destaque para as questões relacionadas à cultura empreendedora - é interessante identificar que as abordagens de design de interfaces interativas podem contribuir para a definição de papéis e de fluxos de trabalho, de maneira a garantir com que os processos tenham uma coerência interna e resultem em transformações reais na empresa.

As abordagens de design de interfaces interativas também podem ser empregadas para fragmentar projetos de grande dimensão como esse em entregáveis menores, de maneira a viabilizar entregas mais pontuais. Dessa maneira, tanto a gestão estratégica quanto a operação acabam sendo beneficiadas. Em vez de tratar da estruturação de uma arquitetura omnichannel de relacionamento, por exemplo, que é um projeto que pode durar anos em contextos como esse, podem ser definidas frentes de trabalho que vão tratar de "especificações funcionais", "requisitos", "arquitetura da informação" e outras etapas menores que, juntas, vão definir a arquitetura omnichannel.

Esse tipo de estratégia pode, inclusive, facilitar com que projetos tão extensos perdurem tempo suficiente para serem concluídos. Com a sua fragmentação em entregáveis menores, é mais fácil otimizar o trabalho e até mesmo reaproveitá-lo, caso os direcionamentos estratégicos sejam alterados no decorrer do processo. Eventualmente, um novo corpo diretor do Sebrae pode não considerar a estratégia omnichannel uma estratégia válida, por exemplo, mas teria insumos e um contexto mais organizado para a implementação de outras estratégias.

\section{Discussão na perspectiva do Design de Interfaces}

A etapa final de análise consistiu na avaliação em conjunto de todos os dados coletados internamente no Sistema Sebrae somada à entrevista realizada com o especialista em design de experiências integradas. Isso permitiu um olhar sistêmico do estudo na mesma medida em que incorporou novas reflexões e uma nova perspectiva de análise.

A contraposição das abordagens de design de interfaces interativas com os documentos identificados no Sebrae apresenta uma possibilidade de como tais abordagens contribuem para a estruturação de uma arquitetura omnichannel de relacionamento. Em ambos os casos, é 
possível identificar que a definição estratégica é o ponto de partida do processo e a materialidade de uma interface, o objetivo final.

No caso do Sebrae, a etapa estratégica caracterizou-se por uma definição macro de como os canais de atendimento e relacionamento devem ser estruturados. Essa definição compreende questões específicas, como o detalhamento de interface de um site ou a redação de scripts de atendimento para uma central de relacionamento. Pelo contrário, o que essa etapa procurou definir foi uma visão genérica sistêmica, capaz de ser moldada para a realidade de cada canal.

A partir dessa definição, seria possível criar um processo padrão que fosse espelhado em cada canal, viabilizando a construção de uma interface específica para cada estrutura e respeitando uma definição macro, que funciona como um fio condutor que garante certa coerência na disposição de todo o ecossistema de canais.

Na prática, esse tipo de abordagem viabiliza que cada decisão seja tomada na etapa coerente ao que ela representa. Nesse momento estratégico, por exemplo, é necessário definir quais tipos de serviço a empresa vai oferecer e quais desses serviços podem ser consumidos apenas por clientes identificados, por exemplo. Tais decisões referem-se ao modelo de negócio da empresa e compreendem decisões de nível estratégico que vão balizar toda a sequência de decisões que devem caracterizar o processo de especificação dos canais (Figura 10).

Figura 10: A macroarquitetura de canais compreende a porção estratégica do processo.

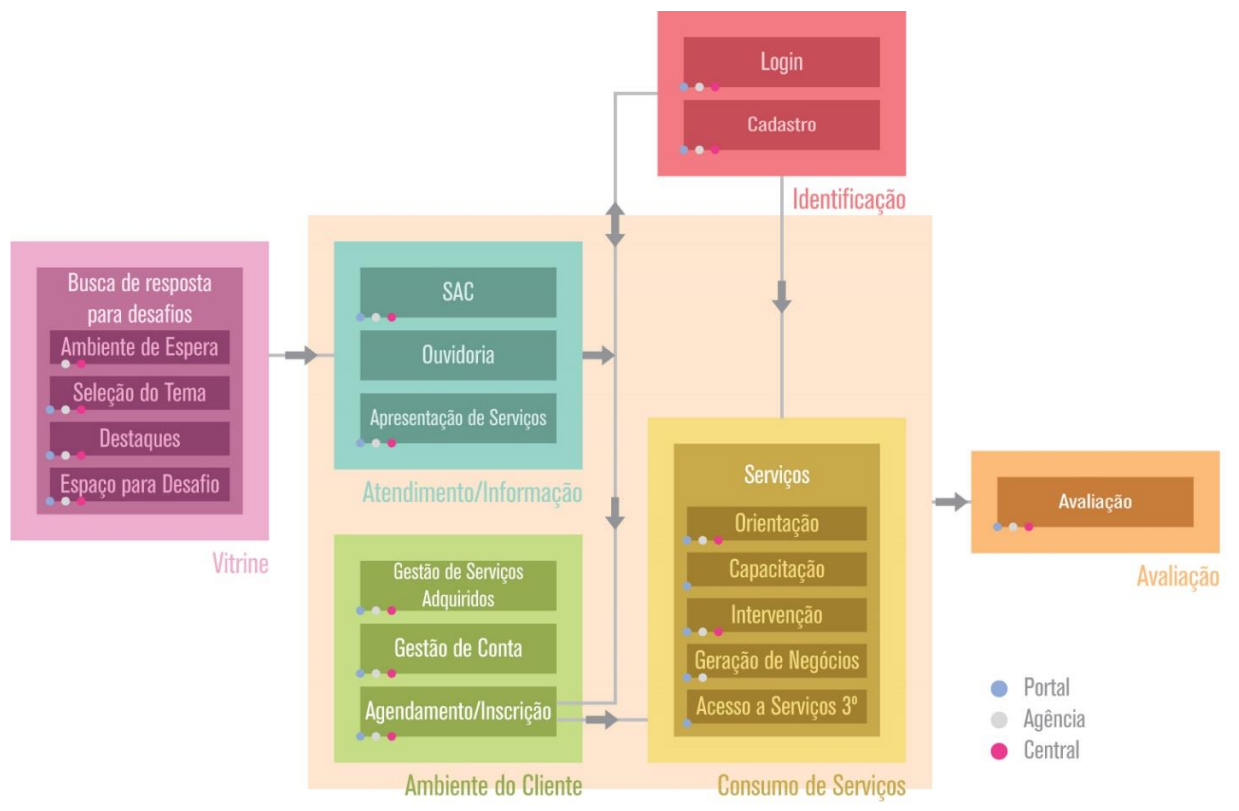

Todavia, mesmo que tal aderência permita o delineamento de um processo lógico e coerente para a definição de uma arquitetura omnichannel de relacionamento, a análise empírica do caso Sebrae permitiu observar outras variáveis que podem interferir diretamente nesse processo e torná-lo ainda mais complexo.

O primeiro ponto refere-se à heterogeneidade que um sistema com tais dimensões pode apresentar. Nesse sentido, não basta operar apenas na integração de diferentes canais, pois é necessário considerar que um mesmo canal pode apresentar conformações tão distintas que se faz necessário categorizá-lo em mais de uma instância. Uma central e relacionamento com 60 colaboradores com formação superior não pode ser tratada da mesma forma que uma central de relacionamento que opera com 4 estagiários, por exemplo. 
Dessa maneira, seria necessário expandir a análise não somente para os tipos de canais existentes, mas também para as diferentes configurações que cada um deles pode assumir, o que pode caracterizá-los como estruturas completamente distintas.

Outro ponto que se tornou evidente nessa análise foi a percepção de uma falta de decisões estratégica claras, seja porque elas de fato não existem, seja porque não são percebidas em todos os níveis hierárquicos da empresa, dado o distanciamento que existe entre as camadas estratégica e operacional.

Nos dois cenários, seria possível empregar abordagens de design de interfaces interativas para contextualizar cada agente envolvido nesse processo. Isso porque, a partir da estrutura em camadas que balizam o desenvolvimento de interfaces interativas, é possível visualizar com maior clareza como os diferentes momentos dessa estruturação se relacionam e como decisões em determinadas etapas impactam em outras.

As abordagens de design facilitam a construção de uma narrativa lógica que parte das definições estratégicas e culminam na especificação das interfaces, permitindo aos participantes do processo que compreendam seu papel em cada uma dessas etapas e a forma como suas ações se relacionam com o contexto.

Essa organização facilita, inclusive, a correlação entre a estrutura dos canais digitais e os requisitos dos sistemas que o suportam, o que trata de um ponto que teve bastante atenção entre os respondentes do questionário e os entrevistados - sistemas tecnológicos integrados.

A partir de definições funcionais claras e do estabelecimento de uma política de navegação entre elas, é viável definir requisitos para os sistemas que vão suportar tais aplicações campos de formulários, fluxos de troca de informações e requisitos de bases de dados. Ainda que não seja possível especificar tecnicamente tais atributos, esse tipo de estrutura subsidia as definições de negócio que se relacionam com os requisitos técnicos.

Por fim, a pesquisa também aponta para a importância de considerar os aspectos pessoais envolvidos em um processo de transformação tão disruptivo como esse. Tal aspecto, referente à cultura organizacional da empresa, foi um ponto de destaque tanto na coleta de dados realizada com os colaboradores do Sebrae, quanto na entrevista realizada com o especialista.

De certa forma, todos evidenciaram a necessidade de trabalhar as mudanças na forma de pensar e agir com relação aos processos da empresa, de maneira a valorizar a viabilizar processos de inovação. Isso extrapola, de fato, as abordagens de design de interfaces interativas, mas constitui um aspecto desse processo que não pode ser ignorado.

Em um contexto tão diverso e com tanta pulverização, não existe processo ou método que suporte a falta de aderência dos próprios colaboradores, independentemente do nível hierárquico em que se encontram. Nesse sentido, as abordagens de design reforçam a característica sistêmica de um processo como esse e que se conclui apenas quando todas as etapas - da estratégia ao detalhamento - são terminadas.

\section{Conclusão}

O estudo buscou investigar as contribuições das abordagens de design de interfaces interativas para a estruturação de uma experiência omnichannel de relacionamento a partir da relação entre o conhecimento teórico existente sobre essas abordagens e a análise de implementação de uma arquitetura omnichannel de relacionamento no Sebrae. Foi possível observar que as abordagens de design de interfaces interativas podem contribuir com um processo de integração de canais de relacionamento na medida em que permitem sistematizar o processo decisório desde as etapas mais genéricas e estratégicas até às etapas mais práticas e tangíveis. Dada a escala e complexidade de um processo de integração de canais de relacionamento estudado, ferramentas que viabilizem a sua visualização são essenciais para garantir uma coerência em todas as suas etapas de execução.

As abordagens de interfaces interativas também contribuem para a modularização de projetos complexos, viabilizando a definição de entregáveis independentes, o que torna todo o 
processo mais ágil e facilita sua execução. Em contextos complexos, é possível que diferentes times estejam envolvidos em diferentes etapas de execução de um projeto, o que demanda métodos que permitam distribuir o trabalho nesse tipo de estrutura.

Todavia, nota-se que esse tipo de abordagem não contempla questões relacionadas ao comportamento dos envolvidos, ou seja, questões de cultura organizacional, e isso pode comprometer e até mesmo inviabilizar a execução de um projeto. Dessa maneira, o processo acaba sendo interpretado como um sistema isolado, imune ao contexto em que está inserido. No caso estudado, em especial, o fator humano talvez tenha sido o elemento mais sensível e, ainda que as questões técnicas pudessem ser relacionadas às referências teóricas de abordagens de design de interfaces interativas, o trabalho poderia tornar-se inviável exclusivamente devido à cultura organizacional. Faz-se necessário, portanto, aprofundar o entendimento de como as relações humanas interferem em um processo de design, de maneira a aproximar as abordagens de design de interfaces interativas ao contexto real onde são aplicadas. Esse ponto se relaciona a abordagens mais contemporâneas de design, com um olhar sistêmico, adaptativo, flexível e indutivo.

O olhar sistêmico diz respeito a tratar as variáveis envolvidas em um processo de design de maneira ampla, considerando o contexto global em que estão inseridas. É necessário ainda que tais abordagens sejam adaptativas e flexíveis para responderem à especificidade de cada caso e também para serem capaz de moldar-se às mudanças promovidas pelo próprio processo de design. Ainda, caracterizam-se como abordagens indutivas por partirem da observação e da exploração de cada situação, para, só então, elaborarem possíveis soluções. O caso apresentado, por fim, reforça a necessidade de abordagens de design dinâmicas e aderentes aos contextos reais e complexos característicos do mundo atual. Dado que o design trata de projetar soluções, é natural que contextos de estruturas físicas e sociais complexas demandem soluções igualmente multifacetadas.

\section{Referências}

Beck, N.; Rygl, D. (2015). Categorization of multiple channel retailing in Multi-, Cross-, and Omni-Channel Retailing for retailers and retailing. Journal of Retailing and Consumer Services. 27, pp.170-178.

Coughlan, A. et al. (2012). Canais de marketing e distribuição. Tradução Lúcia Simonini. 7.ed. São Paulo, Pearson.

Garret, J. (2011). The Elements of User Experience: User-centered design for the Web and Beyond. 2‥ ed. New Riders.

Kotler, P.; Armstrong, G. (2015). Princípios de Marketing. 15ª ed. São Paulo: Pearson.

Mehta, R.; Dubinsky, A.; Anderson, R. (2002). Marketing channel management and the sales manager. Industrial Marketing Management, 31 (5), pp. 429-439.

Neslin, S. et al. (2006). Challenges and opportunities in multichannel customer management. Journal of Service Research 9 (2), pp. 95-112.

Piotrowicz, W.; Cuthbertson, R. (2014). Introduction to the Special Issue: Information Technology in Retail: Toward Omnichannel Retailing. International Journal of Electronic Commerce, v. 18, n. 4, pp. 5-16.

\section{Sobre o(a/s) autor(a/es)}

Henrique Teles da Mota, Mestre, UnB, Brasil <henriquetm@gmail.com>

Tiago Barros Pontes e Silva, PhD, UnB, Brasil<tiagobarros@unb.br> 